\title{
Linfoma de Hodgkin y no Hodgkin durante la gestación: a propósito de dos casos
}

\author{
Beatriz Rojas P. ${ }^{1}$, Matilde Perella A. ${ }^{2}$, Aldonza Ania L. ${ }^{3}$, Teresa Arribas M. ${ }^{1}$, Lorena \\ Guardia D. ${ }^{1}$, Isabel González B. ${ }^{1}$, Belén Carazo H. ${ }^{4}$ \\ ${ }^{1}$ Servicio de Obstetricia y Ginecología, ${ }^{2}$ Servicio de Hematología. ${ }^{3}$ Servicio de Medicina Interna, Hospital de Barbastro, \\ Huesca, España. ${ }^{4}$ Servicio de Obstetricia y Ginecología, Hospital Clínico Universitario Lozano Blesa, Zaragoza, España.
}

\section{RESUMEN}

Las neoplasias hematológicas en el embarazo conllevan un gran riesgo para la madre y el feto. El linfoma, Hodgkin (LH) y no Hodgkin (LNH), es la cuarta causa más frecuente de cáncer diagnosticado en el embarazo. El tipo más frecuente es el LH, mientras que la incidencia de LNH es muy baja. Presentamos dos casos de gestantes de 29 y 25 años, diagnosticadas en el segundo trimestre de la gestación de LNH mediastínico de células $\mathrm{B}$ y LH tipo esclerosis nodular respectivamente. Ambas fueron tratadas con quimioterapia desde el diagnóstico hasta dos semanas antes del parto, con buen resultado perinatal.

\section{PALABRAS CLAVE: Quimioterapia, linfoma de Hodgkin, linfoma no Hodgkin, malformaciones congénitas}

\section{SUMMARY}

Haematological cancer in pregnancy poses a substantial risk to both, mother and fetus. Lymphoma, including Hodgkin's lymphoma $(\mathrm{HL})$ and Non-Hodgkin's lymphoma $(\mathrm{NHL})$, is the fourth most frequent malignancy diagnosed during pregnancy. The most common type of lymphoma in this setting is $\mathrm{HL}$, and the incidence of pregnancy associated NHL is very low. In this report we describe two cases of a 29-year-old woman and a 25-year-old woman both in the second trimester of pregnancy, diagnosed with mediastinal B-cell NHL and nodular-sclerosis HL respectively. They were managed with chemotherapy since the diagnosis as far as two weeks before the delivery, both with a successful fetal outcome.

\section{KEY WORDS: Chemotherapy, Hodgkin's lymphoma, Non Hodgkin's lymphoma, congenital malformations}

\section{INTRODUCCIÓN}

El cáncer se diagnostica en un $0,5-1 \%$ de los embarazos, siendo la segunda causa de muerte materna después de las complicaciones vasculares relacionadas con la gestación (1). Las neoplasias hematológicas, como grupo, representan un $25 \%$ de los cánceres que complican el embarazo, con una incidencia de linfoma de Hodgkin (LH) entre
1:1000 a 1:6000 (2), mientras que la incidencia de linfoma no Hodgkin (LNH) durante el embarazo se ha descrito en 0,8 casos por 100.000 mujeres (3), aunque su prevalencia exacta durante el embarazo es desconocida. Los datos recogidos se refieren a series retrospectivas y descripciones de casos, por lo que es dificultoso establecer unas estrictas guías de manejo.

El objetivo de esta presentación es comunicar 
el manejo de casos de linfoma de Hodgkin y no Hodgkin durante el embarazo.

\section{Casos clínicos}

Caso 1: Linfoma no Hodgkin. Paciente de 29 años, en su 20 semana de gestación tras fecundación in vitro por factor masculino. Ingresa de urgencias a nuestro hospital por disnea de mínimos esfuerzos, ortopnea y masa indurada paraesternal izquierda de aproximadamente $7 \mathrm{~cm}$. Los síntomas habían debutado 2 meses antes y habían sido etiquetados como condritis en otro centro, con aumento progresivo del tamaño del tumor asociado a empeoramiento de la disnea. Entre sus antecedentes personales sólo destacaba una quistectomía por laparoscopia de un quiste endometriósico de ovario izquierdo. Se realizó ecografía que informa de gran masa sólida de $18 \mathrm{~cm}$ que desplaza al ápex cardiaco y se prolonga desde el mediastino anterior, atravesando la pared torácica y abombando la piel. La biopsia informa de linfoma no Hodgkin de células grandes tipo $B$ (Figura 1). La resonancia nuclear magnética que se planteó para etapificación completa del tumor no pudo ser realizada por intolerancia de la paciente al decúbito, debida a la intensa ortopnea, pero se interpreta como estadio IIB. Después de un consenso multidisciplinar y revisando bibliografía, se comenzó tratamiento con dosis estándar de régimen quimioterápico R-CHOP (rituximab, ciclofosfamida, adriamicina, vincristina y prednisona) cada tres semanas, por un total de seis ciclos. El tratamiento comenzó a las 21 semanas de gestación y el último ciclo a las 33 semanas, objetivándose una disminución progresiva de la masa hasta los $3 \mathrm{~cm}$. Por el riesgo aumentado de retra- so de crecimiento intrauterino y parto pretérmino, realizamos un seguimiento ecográfico cada 3 semanas que incluía medición de la longitud cervical vía transvaginal. A las 35,6 semanas de gestación (20 días tras el quinto ciclo de quimioterapia) se llevó a cabo la inducción de parto que terminó en parto eutócico de un recién nacido sano de 2.200 g, Apgar 9/10. Fue recogida la sangre de cordón umbilical para reservar células madre en el caso de que la paciente necesitara un autotrasplante de médula ósea, y la placenta se envío a Anatomía Patológica para descartar enfermedad metastásica en la misma. Se da de alta a la paciente cinco días postparto, tras completar el último ciclo de quimioterapia. Un mes después del parto se administra quimioterapia de segunda línea por respuesta parcial y a pesar de otras dos líneas de tratamiento más, la enfermedad continua en progresión al año del diagnóstico y se decide tratamiento paliativo.

Caso 2: Linfoma de Hodgkin. Paciente de 25 años, primigesta, que acude a nuestro hospital refiriendo una masa en región cervical izquierda y otra de similares características en región inguinal izquierda desde la quinta semana de gestación, con aumento progresivo de tamaño. No presenta antecedentes de interés previos. Se realiza biopsia-exéresis de adenopatía inguinal bajo anestesia local y de forma ambulatoria con resultado histológico de linfoma de Hodgkin tipo esclerosis nodular (Figura 2). Basándonos en la exploración física, se clasifica como un estadio IIIA y se discuten las diferentes opciones de tratamiento. Después de consenso multidisciplinar, la paciente comienza tratamiento con dosis estándar de adriamicina, bleomicina, vinblastina y dacarbacina (ABVD), administradas cada dos semanas, por un total de seis ciclos. El tratamiento

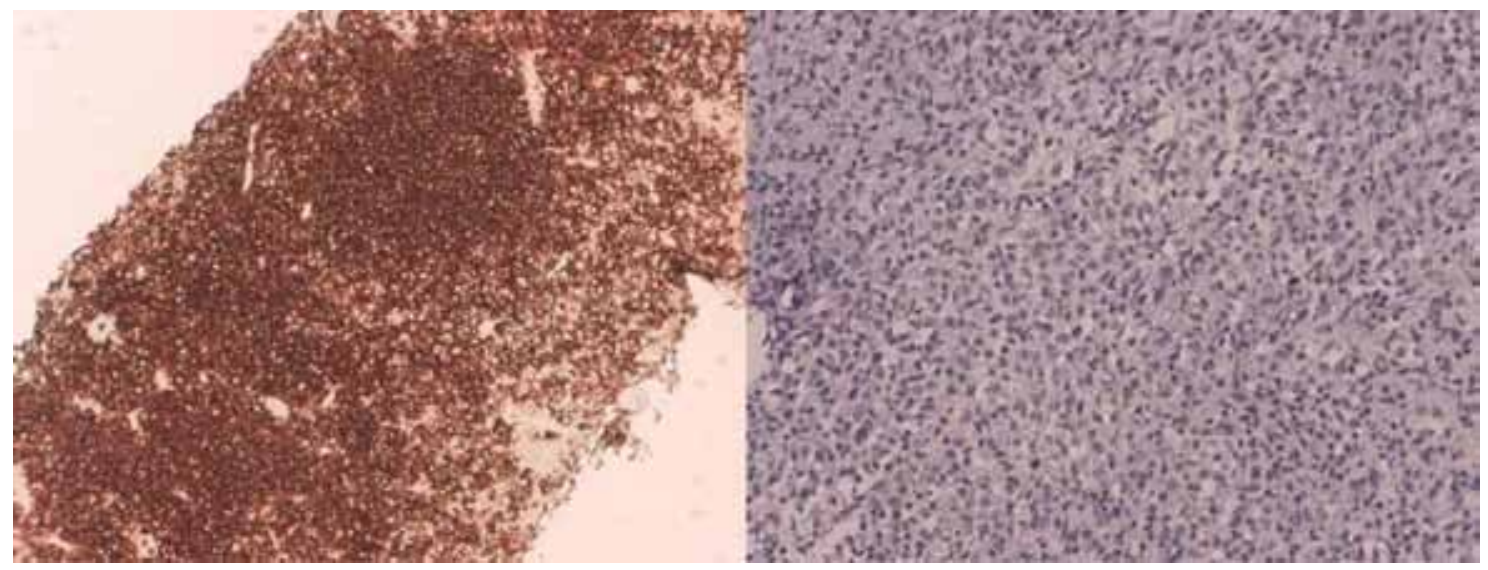

Figura 1. Linfoma no Hodgkin. Con tinción de hematoxilina-eosina a 20 aumentos proliferación linfoide difusa con célula pequeña y grande. Con tinción inmunohistoquímica CD 20 a 10 aumentos, positividad difusa e intensa en la membrana. 


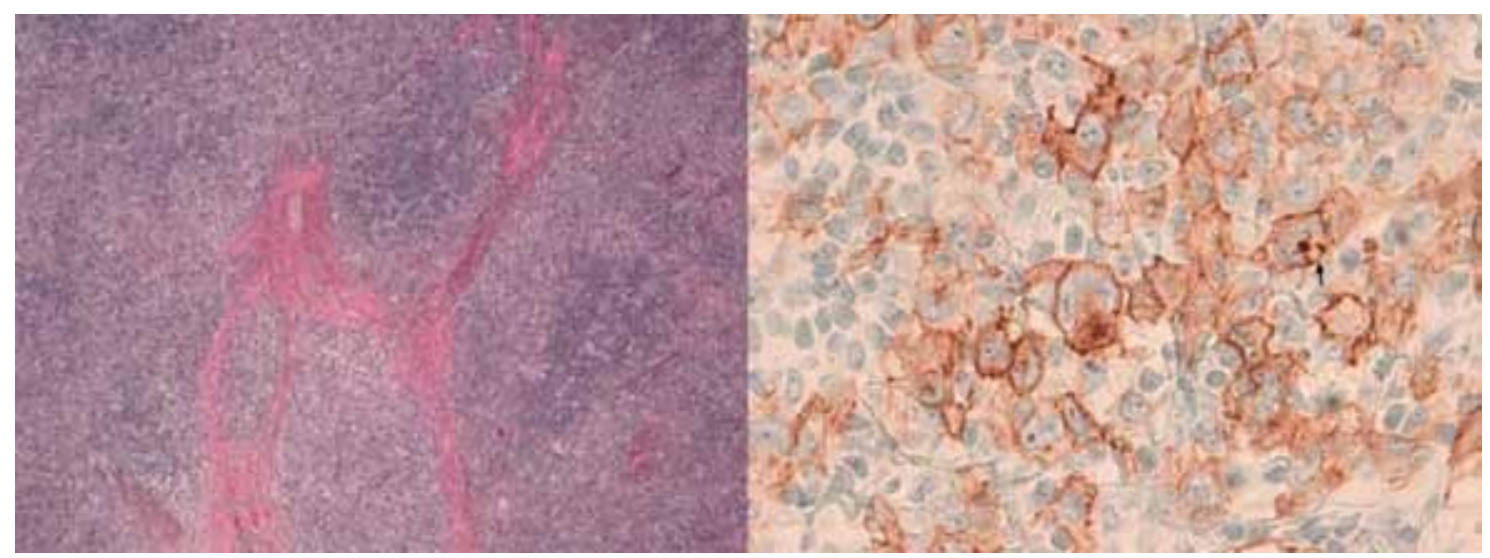

Figura 2. Linfoma de Hodgkin. Con hematoxilina-eosina a 4 aumentos, ganglio linfático desestructurado con tractos fibrosos y celularidad atípica. Con inmunohistoquímica a 63 aumentos, CD 30 positiva en membrana y en Golgi en células grandes (flecha).

comenzó a las 23 semanas de gestación y el último ciclo a las 33 semanas. Se completó la quimioterapia sin efectos secundarios graves salvo astenia. A las 36 semanas de gestación, tres semanas tras el último ciclo, se indujo el parto que terminó en parto vaginal obteniéndose un recién nacido varón, sano, de $2.580 \mathrm{~g}$, Apgar 9/10. El recién nacido no presentó signos de mielosupresión y fue dado de alta en buenas condiciones a los dos días de vida. Se aconsejó a la madre inhibir la lactancia. Una semana después del parto, se administró el cuarto ciclo de quimioterapia hasta completar seis ciclos. Cuatro meses posparto, se observa progresión de la afectación nodal por lo que se decide tratamiento quimioterápico de segunda línea, encontrándose en tratamiento en el momento de la publicación.

\section{DISCUSIÓN}

El diagnóstico de neoplasias hematológicas es dificultoso durante la gestación porque muchos de sus síntomas pueden confundirse con síntomas habituales del embarazo. Estas pueden ser las causas del retraso en el diagnóstico, como fue en nuestros dos casos. La biopsia o exéresis de la masa o adenopatías no se asocia con riesgo fetal o materno aumentado ya que puede realizarse con anestesia local o general, sin problemas (1). La ecografía o la resonancia nuclear magnética nos pueden llevar al diagnóstico sin que conlleven un aumento del riesgo de malformaciones fetales, sin embargo la tomografía axial computarizada debe ser evitada en el embarazo (4). La gran mayoría de los casos de LH y LNH se tratan inicialmente con quimioterapia, independiente del estadio al diagnóstico, por lo que el estadiaje de las pacientes embarazadas con linfoma debe ser limitado y basarse en la historia clínica, el examen físico, los análisis de sangre de rutina, la biopsia de médula ósea y las radiografías con protección abdominal (5).

Basándonos en los resultados recogidos ampliamente en la literatura, las pacientes con LH en el embarazo deben ser tratadas con el régimen ABVD mientras que en las pacientes con LNH el tratamiento de elección es R-CHOP, considerados ambos seguros en segundo y tercer trimestre $(4,5)$. Revisando la evidencia científica, la seguridad fetal de la quimioterapia en el primer trimestre es limitada por lo que, si se diagnostica $\mathrm{LH}$ avanzado o LNH agresivo antes de las 14-16 semanas, debe considerarse la interrupción de la gestación, seguido de un completo estadiaje y adecuadas dosis de quimioterapia $(5,6,7)$.

El linfoma no Hodgkin de células grande B difuso, es más agresivo y puede causar complicaciones que potencialmente pueden comprometer la vida de la paciente. Debido a que su pronóstico es malo y conlleva rápida progresión, es necesario tratarlo con regímenes de quimioterapia combinados (8). El régimen $\mathrm{R}-\mathrm{CHOP}$ ha demostrado ser superior que el CHOP aislado y comparable con regímenes de quimioterapia más agresivos en el tratamiento de pacientes embarazadas con LNH, con tasas de curación del $82 \%$ y tasas de supervivencia a los cinco años del 89\% (9).

El tratamiento del LH normalmente conlleva entre dos y seis ciclos de ABVD. Hay muchas series de casos retrospectivas de seguimiento de pacientes que han seguido este tratamiento en los tres estadios durante el embarazo sin que se hayan publicado casos de anomalías congénitas o psicológicas en el seguimiento de estos pacientes (10).

Otro riesgo potencial para el feto es la transmisión materno-fetal de células malignas y la afectación 
de la placenta. Ambas son extremadamente raras en el embarazo pero hay varios casos descritos en el LNH $(11,12)$. En nuestros casos las placentas fueron estudiadas histológicamente, sin hallarse evidencia de metástasis.

El parto debe ser pospuesto hasta tener seguridad de la madurez pulmonar fetal, sin comprometer la salud de la madre ni del feto. La cesárea de entrada no está recomendada, salvo que haya otra causa obstétrica, ya que el estadiaje por laparotomía no es necesario (13). El parto debe ser planeado no antes de dos semanas tras el último ciclo pero no más tarde de 3-4 semanas para permitir la recuperación del recuento sanguíneo en la madre y el feto $(2,14)$. Debe recogerse sangre de cordón umbilical como fuente potencial de células madres HLA compatible con el progenitor, en caso que fuera necesario un trasplante de médula ósea (2).

Aunque los datos sobre la mayoría de los agentes quimioterápicos y la lactancia materna son escasos, se recomienda inhibir la lactancia durante la quimioterapia (2).

\section{CONCLUSIÓN}

En general, la mayoría de las pacientes gestantes diagnosticadas de una neoplasia hematológica tienen buena evolución del embarazo y su pronóstico no difiere significativamente de no gestantes. Las dosis estándar de quimioterapia están recomendadas en el embarazo. Son necesarios más datos recogidos prospectivamente y se requiere la colaboración internacional para establecer guías de tratamiento.

\section{REFERENCIAS}

1. Brenner B, Avivi I, Lishner M. Haematological cancers in pregnancy. Lancet 2012;379:580-87.

2. Hurley $\mathrm{T}$, McKinell J. Hematological malignancies in pregnancy. Obstet Gynecol Clin N Am 2005;32:595-614.

3. Kuzel TM, Benson AB. Non-Hodgkin's lymphoma. In: Gleicher N, editor. Principles and practi- ce of medical therapy in pregnancy. 2nd edition. East Norwalk (ct): Appleton and Lang; 1992. p. 1078-81.

4. Vandenbriele C, Dierickx D, Amant F, Delforge $M$. The treatment of hematologic malignancies in pregnancy. F,V\&V ObGyn;2010;2:74-87.

5. Pereg $D$, Koren $G$, Lishner $M$. The treatment of Hodgkin's and non Hodgkin's lymphoma in pregnancy. Haematologica 2007;92:1230-7.

6. Cannellos GP, Anderson JR. Chemotherapy of advanced Hodgkin's disease with MOPP, ABVD or MOPP alternating with ABVD. N Engl J Med 1992;327:1478-84.

7. Lees C, Tsirigotis J, Carr J. T cell non-Hodgkin's lymphoma presenting in the first trimester of pregnancy. Postgrad Med J 1994;70:371-2.

8. Cetin O, Aydogan B, Uludag S, Sen C. Non Hodgkin lymphoma diagnosed during pregnancy: a case report. Periant J 2011;19:94-8.

9. Pérez C, Amin J, Aguina L. Primary mediastinal large B-cell lymphoma during pregnancy. Case Rep Hematol 2012; 2012:197347

10. Hodby K, Fields PA. Management of lymphoma in pregnancy. Obstet Med 2009;2:46-51.

11. Viñuela $C$, Muñoz A, Pérez R, García $P$, La Cruz a, Ortiz L. A case of primary mediastinal B-cell lymphoma diagnosed during cesarean section: a rare presentation. European $\mathrm{J}$ Clin Med Oncol 2010;2:1.

12. Corredor E. Cancer in pregnant women and its extension to fetoplacental unit. Rev Colomb Obstet Ginecol 2007;58:232-36.

13. Rizack T, Mega A, Legare R. Management of hematological malignancies during pregnancy. Am J Hematol 2009;84:830-41.

14. Vandenbriele C, Vassou A. Pentheroudakis G, Van Calsteren K, Amant F. Hematologic Malignancies in Pregnancy. In: Acute Leukemia - The Scientist's Perspective and Challenge. Mariastefania Antica (Ed). 2011. Disponible en: http:// www.intechopen.com/books/acute-leukemiathe-scientist-s-perspective-and-challenge/hematologic-malignancies-in-pregnancy. 